\title{
SERÁ A INVESTIGAÇÃo SOBRE ALTERAÇÕES CLIMÁTICAS VERDADEIRAMENTE COLABORATIVA?
}

\author{
Sofia Bento, Marta Varanda, Audrey Richard-Ferroudji \& Nicolas Faysse
}

\begin{abstract}
RESUMO
O artigo centra-se no estudo da interação e da troca de conhecimento entre cientistas e stakeholders na investigação para adaptação às alterações climáticas. Baseia-se numa investigação realizada no contexto de um programa Europeu denominado Circle Era-Net delineado para impulsionar a ligação entre cientistas e stakeholders, através de uma rede de pesquisa europeia que estuda as alterações climáticas. Foram entrevistados 33 cientistas, stakeholders e financiadores envolvidos em sete projetos apoiados pelo programa Circle-Med. A colaboração e a troca de conhecimentos constituíram intenções reconhecidas, mas não foram práticas observáveis nas atividades promovidas pelas várias equipas de investigação. A partir destes resultados, defende-se serem necessárias melhorias que permitam alcançar uma ciência verdadeiramente colaborativa entre cientistas e stakeholders. Neste sentido, o artigo apresenta algumas sugestões relativas à conceção de novos critérios para as chamadas de projetos de investigação, assim como à monitorização e à avaliação do processo colaborativo numa pesquisa científica e à integração das Ciências Naturais e sociais na investigação sobre alterações climáticas.
\end{abstract}

Palavras-Chave

Colaboração; interação; stakeholders; cientistas; alterações climáticas

\section{INTRODUÇÃo}

As alterações climáticas constituem hoje um tópico incontornável na agenda das sociedades contemporâneas, uma vez que trazem mudanças globais que afetam as paisagens, as economias e as sociedades. Há, por isso, uma crescente necessidade de políticas ambientais, a nível nacional e internacional, para lidar de forma incisiva com esta questão.

As alterações climáticas são, também, um exemplo paradigmático do papel do conhecimento científico na sociedade actual, principalmente nas políticas ambientais. Por um lado, existem muitas provas científicas sobre fenómenos climáticos e muito do conhecimento relativo a estes fenómenos encontra-se compilado e acessível (IPPC, 2007). Existem hoje provas concretas de que as alterações climáticas constituem uma realidade séria e que estão relacionadas com o efeito de estufa (PNUD, 2007-2008). Os governos também aceitam esta evidência científica. Por outro lado, a complexidade e a incerteza são duas características do conhecimento existente sobre as alterações climáticas. Por esta razão, há ainda um longo caminho a percorrer antes de podermos fazer avaliações acertadas sobre o impacto das alterações climáticas e consequentes custos a nível local, nos vários países (Santos, 2012). Acima de tudo, as alterações climáticas continuam a ser um assunto de profunda controvérsia científica (Latour, 2014) que, por 
vezes, instiga a necessidade de a comunidade climatologista traduzir e simplificar a informação (Schiermeier, 2007).

De facto, a alterações climáticas têm estado cada vez mais associadas a questões que saem da esfera da natureza para penetrar no domínio da política, da governança e da constituição da sociedade. Neste contexto, políticos e cientistas assumiram a liderança das questões em torno das alterações climáticas. Contudo, há ainda uma grande distancia entre a comunidade científica e a sociedade civil relativamente a este assunto (Grundmann \& Stehr, 2010; Comissão Europeia, 2014).

Além da estratégia de mitigação, as sociedades têm lidado com as alterações climáticas centrando-se, cada vez mais, na adaptação local. Por um lado, a colaboração, a troca de conhecimentos e a integração de medidas têm sido consideradas como pilares essenciais para uma adaptação às alterações climáticas bem sucedidas (Wilby \& Dessai, 2010). Por outro, as agências financiadoras e as instituições públicas e privadas estão agora muito interessadas em soluções de adaptação baseadas na participação da sociedade. Para responder a este novo repto, a comunidade científica precisa de ajustar as suas práticas, o que implica inovar no processo de conceção da pesquisa e na produção de conhecimento, introduzindo os stakeholders numa fase mais precoce do processo de investigação (Eakin et al., 2007).

O presente artigo interessa-se pela forma como a interação entre cientistas e stakeholders se desenvolve no contexto de um programa concebido para apoiar a investigação para a adaptação às alterações climáticas. Mais especificamente, a pesquisa insere-se no programa Europeu denominado "Circle ERA-NET"', uma plataforma do sétimo programa quadro de apoio à investigação, dedicada à coordenação de políticas científicas de países europeus para a adaptação às alterações climáticas². O programa foi desenvolvido para promover a investigação europeia nesta matéria e impulsionar a ligação entre cientistas e stakeholders. As várias atividades do programa Circle, as chamadas para investigação, as redes de equipas de investigação ou as plataformas de transferência de conhecimento foram definidas de modo a contribuir com novas formas de partilha e de disseminação de conhecimentos para a ação dos decisores políticos.

Assim, na investigação que sustenta o presente artigo analisam-se projetos científicos financiados por este programa, de forma a compreender como os objetivos iniciais e formais do programa foram efetivamente alcançados e, simultaneamente, a recomendar medidas que fomentem a colaboração e a interdisciplinaridade na investigação sobre as alterações climáticas.

Oficialmente, o programa CIRCLE foi lançado para criar uma comunidade de investigação na região do Mediterrâneo através de programas de investigação colaborativos versando sobre o impacto das alterações climáticas, com o objectivo de levar os resultados desta investigação aos atores, em particular aos decisores políticos. O programa de investigação que tomamos como objeto de estudo para este artigo designa-se "Zonas

\footnotetext{
' O acrónimo "Circle" significa "Climate Impact Research \& Response Coordination for a Larger Europe".

${ }^{2}$ Para mais informação, é importante aceder ao sitio eletrónico: www.circle-era.eu.
} 
Costeiras Integradas e Gestão da Água" e incluiu países não europeus situados na orla da bacia do Mediterrâneo. O Circle Med foi apoiado por agências de financiamento de França, Galiza (Espanha), Itália, Israel e Portugal. A primeira chamada para estes projetos realçava a necessidade de uma gestão integrada da água e das zonas costeiras, em virtude da escassez hídrica no Mediterrâneo. Como mencionado no anúncio da chamada, as propostas para projetos deveriam criar novos conhecimentos relativamente a "estratégias adaptadas no setor da água e das zonas costeiras" (Circle-Med, 2007).

A chamada de propostas consiste num documento de 4 páginas, dividido em três partes lançado em 2007. Na introdução, a área do Mediterrâneo é apresentada como objeto da investigação, por constituir uma região com problemas climáticos manifestos em alterações da temperatura, da precipitação, do nível do mar, e em fenómenos climáticos extremos. Neste documento, as conclusões científicas do IPPC (2007) são usadas precisamente para justificar a necessidade de serem realizados estudos de impacto e planos de adaptação na região. A segunda parte apresenta o contexto da chamada, baseada nos seguintes pontos: i) nas especificidades das regiões do mediterrâneo; ii) nos recursos hídricos nas regiões do Mediterrâneo e; iii) nas zonas costeiras. A terceira parte consiste nas duas orientações do anúncio do concurso que visam nomeadamente: i) estratégias de adaptação no setor da água e zonas costeiras que foquem pontos como zonas urbanas, agricultura, indústria, turismo, ecossistema costeiro e gestão dos recursos de água e ii) um novo equilíbrio na gestão integrada dos recursos de água e recursos costeiros com um enfoque na racionalização, na governança da água, na regulação entre regiões, e no planeamento de ações inovadoras.

A chamada para propostas era ambiciosa. Frisava a importância de "um novo equilíbrio na gestão integrada dos recursos hídricos e das zonas costeiras", nomeadamente nas formas de racionalizar e governar diferentes interesses nos usos da água na perspetiva das alterações climáticas. Referia, igualmente, que a "multidisciplinaridade devia desempenhar um papel importante nos diferentes campos de investigação" e que era desejado "um bom equilíbrio entre as ciências biotécnicas (da hidrogeologia à agronomia) e as Ciências Sociais". Além do mais, sublinhava que "as estratégias de adaptação exigiam uma colaboração precoce com os decisores, com vista a difundir de forma eficaz as recomendações dos projetos aos agentes da administração. Os projetos de investigação deviam identificar e fornecer informações para resolver problemas práticos de adaptação (Circle-Med, 2007). O nosso projeto envolveu entrevistas através das quais questionámos sobre a concretização destes objetivos, assim como acerca da forma como teriam influenciado os conteúdos dos projetos e como estes teriam resolvido iniciar um diálogo com a sociedade. Procurou-se fazer a análise da interação entre a ciência e a prática, sendo que esta inclui o modo como os cientistas, stakeholders, decisores e outros intervenientes, trocam ideias e informação (Groot, Hollaender \& Swart, 2014). Na investigação foi nossa intenção entender melhor que tipo de intervenientes - não-cientistas - estiveram envolvidos nos projetos do Circle-Med, com que objetivo e de que modo. Desta forma, durante o estudo: i) classificámos os stakeholders e as intenções das equipas nas suas iniciativas para dialogar com os stakeholders; ii) analisámos o 
teor das interações entre cientistas e não-cientistas (conversas cara-a-cara e discussões informais, questionários e workshops) e iii) investigámos os meios e níveis de interação, bem como a forma como os resultados e dados científicos eram comunicados. Nos pontos seguintes apresentaremos uma síntese da teoria existente acerca dos processos de (co)produção de conhecimento, os stakeholders e a sua colaboração na investigação. Nos dois últimos pontos explicamos a metodologia seguida, assim como os principais resultados da investigação conduzida.

\section{Produção de ConheCimento COM A COlaboraÇÃo de STAKeHolders}

Durante a última década, a literatura sobre produção de conhecimento científico (sociologia da ciência, estudos sociais da ciência e estudos sobre inovação) enfatizou, de forma crescente, o papel da sociedade civil, da administração pública e de empresas privadas na produção de conhecimento e na análise do seu impacto na sociedade. A ciência modo 2 (Nowotny, Scott \& Gibbons, 2003) configura uma forma de concetualização deste novo modo de produção de conhecimento: significa que os progressos científicos ultrapassam as fronteiras disciplinares e, simultaneamente, são criados em vários espaços, deixando de estar confinados ao espaço dos laboratórios e dos centros de investigação. Um resultado deste novo paradigma sobre o modo como "fazer ciência", é o aumento do número dos intervenientes na produção científica. Também começaram a ser desenvolvidas novas formas de organização e de comunicação entre os atores científicos e os não-científicos. No fundo, o objetivo da produção científica está agora menos centrado no avanço do conhecimento e mais na resolução de problemas. Noutras abordagens sobre a produção de ciência, tais como as da ciência pós-normal (Funtwovics \& Ravetz, 1990), os stakeholders ganham um novo papel, motivado pelas incertezas inerentes aos problemas científicos que também trazem à discussão a interrogação sobre novos valores.

O papel atribuído a esses intervenientes não se prende apenas com uma maior democratização do processo científico ou com as questões da interação entre a comunidade científica e a sociedade civil. Segundo Ravetz (1999), a participação pública e o envolvimento dos stakeholders são elementos chave para se obter conhecimentos científicos de qualidade e que possam ser aplicados na prática. A inclusão de não-cientistas no processo de investigação sobre questões ambientais era já considerada importante no séc. XIX. Por exemplo, Stephen Forbes (1844-1930) foi um naturalista americano que escreveu um ensaio intitulado "The Lagoon as microcosm: Doing field research in lagoons in the Illinois State". Neste artigo (Forbes, 1925), o autor mostrou como os recursos naturais estavam ligados ao conhecimento local, aos stakeholders e aos problemas ambientais mais amplos (Schneider,2000). A importância do conhecimento local foi também demonstrada em estudos sociais da ciência (Irwin, 1995; Wynne, 1996). As Ciências Naturais têm tido cada vez mais em consideração estes intervenientes e os seus conhecimentos (Hoverman et al.,2011; Hulme et al., 2009; Kuper et al., 2009; Roncoli, 2007).

Entende-se o conceito de stakeholder em sentido lato, como alguém com interesse ou com algum tipo de preocupação (pode ser quem afeta ou quem é afetado num 
determinado processo). A literatura mostra dois tipos de abordagens para a definição de stakeholder (Reed et al., 2009): uma abordagem mais restrita que limita os stakeholders a intervenientes com poder ou interesses considerados "legítimos". Esta abordagem é usada essencialmente pela gestão mas tem sido questionada, em particular no que respeita ao conceito de "legitimidade de interesses". A outra abordagem alarga a noção de stakeholder a entidades que possam ser afetadas pelo desempenho organizacional. Tal inclui pessoas atingidas, mas também realidades menos identificáveis, como o futuro das entidades não humanas e/ou o bem estar das gerações futuras. $\mathrm{Na}$ área dos recursos naturais, Coase (citado em Reed et al., 2009, p.1934) integra no grupo dos stakeholders, tanto os poluidores como as vítimas, uma vez que os primeiros podem afetar o ecossistema poluindo o ambiente, e os segundos podem ser, ou não, afetados pela poluição causada. Neste contexto, os stakeholders chave podem ser agricultores ou outros utilizadores de recursos naturais, agentes de desenvolvimentos, decisores, responsáveis da administração pública, de empresas ou de organizações não-governamentais (Grimble, 1998). Neste estudo, e para simplificar, vamos tipificar os stakeholders em dois tipos: 1) administração pública (local ou regional) e 2) intervenientes locais, que podem incluir intervenientes económicos (pescadores, agricultores e comércio), associações locais e também quem utiliza os recursos com outros fins, por exemplo, o lazer.

O envolvimento dos stakeholders na investigação é um tema que faz parte de uma discussão mais ampla sobre a questão da produção de investigação e a possível articulação entre ciência e sociedade. Os conceitos de colaboração e de investigação participada são hoje em dia cruciais aos novos processos de fazer ciência. A investigação colaborativa e o envolvimento dos stakeholders podem facilitar a transferência de resultados científicos para as políticas e a prática, em geral.

O envolvimento do stakeholder é descrito como um processo que garante a identificação e a participação das pessoas chave no processo de investigação. Estas podem, assim, influenciar o desenrolar da investigação e usar os resultados desta. Lang e outros (2012) definem a investigação transdisciplinar como um processo interativo e participativo que se inicia com a identificação de um problema comum, depois resolvido por um coletivo de intervenientes (investigadores e não investigadores) partilhando a mesma linguagem. Este processo iterativo que reúne os vários stakeholders tem o potencial de conduzir a uma solução consensual para o problema, facilitando a reintegração do conhecimento na ciência e na sociedade. Os autores citam diversas vantagens deste envolvimento dos stakeholders: a possibilidade de lidar com problemas complexos e graves (Reed, 2008), a hipótese de aprender com os problemas e o próprio processo de investigação (Lovens et al., 2015) e a possibilidade de aplicar o conhecimento à prática garantindo-se a transferência dos resultados científicos (Cohen, 1997) ${ }^{3}$.

\footnotetext{
${ }^{3}$ Contudo, a incorporação deste tipo de relação no processo de investigação ainda não é um procedimento corrente. Também não é um processo linear. No contexto da investigação europeia, e no caso específico da rede Circle, foi recentemente redigida um manual de procedimentos que resulta das várias experiências entre parceiros, investigadores e stakeholders e que tem o propósito de ser usado na orientação de uma política de investigação dirigida à adaptação às alterações climáticas (Groot, Hollaender \& Swart, 2014).
} 
Porém, os resultados da interação podem variar significativamente. O grau de envolvimento e o tipo de stakeholder também podem ser distintos, dependendo do tipo de investigação que está a ser levada a cabo, e ainda, dependendo do ponto em que essa investigação se encontra. Neste sentido, o próprio conceito de colaboração tem de ser usado de forma cautelosa. Katz e Martin (1997) chamaram a atenção para o facto de a colaboração respeitar uma realidade multidimensional, uma característica à qual não tem sido dada a devida atenção. No artigo que citámos, o conceito de colaboração é entendido exclusivamente como relação entre investigadores. Voltando ao objeto que nos interessa, a colaboração entre investigadores e stakeholders pode apresentar formatos múltiplos. Para finalizar, também nos parece pertinente haver reflexão sobre o facto de este tipo de colaboração poder traduzir-se em obstáculos e custos para ambos os lados.

\section{Metodologia E PROJETOS DE INVESTIGAÇÃo ESTUdAdoS}

O artigo reflete o estudo que efetuámos aos projetos do Programa de Investigação Circle, depois de estes estarem concluídos. Além de outra informação que recolhemos sobre os contextos dos projetos, realizámos 33 entrevistas: 21 a cientistas, 9 a stakeholders e 3 a financiadores. Conduzimos também 3 entrevistas a pessoas que estavam na gestão do programa, de forma a reunir informação sobre os objetivos e a história do programa do Circle Med. Neste grupo estiveram um antigo responsável pelo departamento de investigação do Ministério do Ambiente Francês, um membro do comité científico do programa Circle e uma responsável pela coordenação do programa nos diversos países. A maior parte das entrevistas foi realizada presencialmente. Apenas 8 foram realizadas ao telefone ou por correio eletrónico. Todas as entrevistas realizadas foram gravadas e transcritas.

Os investigadores e stakeholders entrevistados foram convidados a refletir e a partiIhar as experiências da investigação e a sua ligação com os outros intervenientes. Embora as nossas entrevistas fossem estruturadas, tornou-se por vezes difícil seguir o guião, pois os investigadores ou não se sentiam sempre motivados para discutir os tópicos propostos ou preferiam evitar o assunto porque não dispunham de conhecimentos precisos nesse âmbito. Os investigadores preferiam, aliás, com alguma frequência, expor os seus pontos de vista de forma muito pessoal, pelo que, nestes casos, seguimos os seus discursos. Usámos dois modelos de guião de entrevista: uma versão dirigida aos coordenadores e investigadores envolvidos e outra versão dirigida aos stakeholders. A tabela que se segue mostra os principais tópicos das entrevistas (Tabela 2). As linhas condutoras de cada entrevista decorreram das especificações do projeto em questão.

As entrevistas com os investigadores realizaram-se entre Maio de 2012 e Junho de 2013. Analisámos 7 projetos de investigação financiados pelo Circle-Med e 21 equipas de investigação (Portugal, França, Itália, Israel, Tunísia, Croácia e Marrocos). Um dos projetos, denominado Aquimed, foi excluído desta análise, uma vez que os investigadores deste projeto eram também investigadores do presente estudo. Além desta, recolhemos informação secundária proveniente dos relatórios científicos e da produção científica de cada projeto. 


\begin{tabular}{ll}
\hline \multicolumn{1}{c}{ INVESTIGADORES } & \multicolumn{1}{c}{ STAKEHOLDERS } \\
\hline Caracterização do investigador & Caracterização do stakeholder \\
\hline $\begin{array}{l}\text { Objetivos da investigação relativa- } \\
\text { mente à interação com o stakeholder }\end{array}$ & $\begin{array}{l}\text { Critério para seleção de stakehol- } \\
\text { ders/formas de recrutamento de } \\
\text { stakeholders para o projecto }\end{array}$ \\
\hline $\begin{array}{l}\text { Caracterização da interação e da } \\
\text { colaboração com o stakeholder }\end{array}$ & $\begin{array}{l}\text { Atual colaboração dos stakeholders } \\
\text { com a equipa de investigação }\end{array}$ \\
\hline $\begin{array}{l}\text { Atual cooperação entre stakehol- } \\
\text { ders e investigadores }\end{array}$ & $\begin{array}{l}\text { Classificação da relação entre stakehol- } \\
\text { ders e equipa de investigadores }\end{array}$ \\
\hline $\begin{array}{l}\text { Organização de workshops participati- } \\
\text { vos e/ou atividades de disseminação }\end{array}$ & $\begin{array}{l}\text { Participação em workshops e/ou ati- } \\
\text { vidades de disseminação }\end{array}$ \\
\hline $\begin{array}{l}\text { Impacto das conclusões cientí- } \\
\text { ficas nas políticas do setor }\end{array}$ & $\begin{array}{l}\text { Participação nas elaboração de conclu- } \\
\text { sões e/ou atividades de disseminação }\end{array}$ \\
\hline
\end{tabular}

Tabela 1: Descrição dos tópicos contemplados nas entrevistas

As categorias usadas para analisar a interação e os tipos de ligação entre investigadores e stakeholders foram inspiradas em Phillipson e outros (2012). Estes autores utilizam uma grelha de categorias para analisar um programa específico sobre Economia Rural e Utilização do Solo, que levou a cabo uma investigação interdisciplinar. Esta greIha contemplava sete categorias relativas ao envolvimento dos intervenientes externos: quem respondeu aos inquéritos, participantes nos eventos, membros de comissões de aconselhamento ou de direção, parceiro do projeto, consultor, cliente da investigação e/ ou visitante no projeto. Os responsáveis pediam, então, para que os seus interlocutores indicassem a natureza do envolvimento dos stakeholders nos projetos de investigação. Poderiam escolher entre nove tipos de contributos possíveis: contribuir para o enquadramento do problema; facilitar o acesso a equipamento de investigação, a materiais ou estudos de estudo; contribuir para a discussão sobre o desenho do projeto; contribuir na produção de conhecimento enquanto parte igual; fornecer informações ou opiniões enquanto sujeitos da investigação; ajudar na recolha de dados; rececionar os resultados das investigações; contribuir com reações sobre os resultados obtidos e ajudar na disseminação dos dados. Uma vez que nos casos que estudámos os padrões de relação entre cientistas e outros intervenientes eram simples, a grelha baseou-se no processo de produção científica e nos mecanismos de colaboração escolhidos por cada projeto.

A figura 1 apresenta informação acerca dos projetos Circle analisados na presente pesquisa, indicando os países coordenadores, o número de parceiros e a localização dos estudos de caso envolvidos.

Podemos observar a existência de 4 projetos coordenados por equipas italianas, 2 projetos coordenados por equipas francesas e 2 projetos coordenados por equipas portuguesas. Também se observam estudos de caso nos vários países do Mediterrâneo não pertencentes à União Europeia como Marrocos, Tunísia e Israel. Todos os projetos iniciados a partir de 2010 foram concluídos em 2012. 


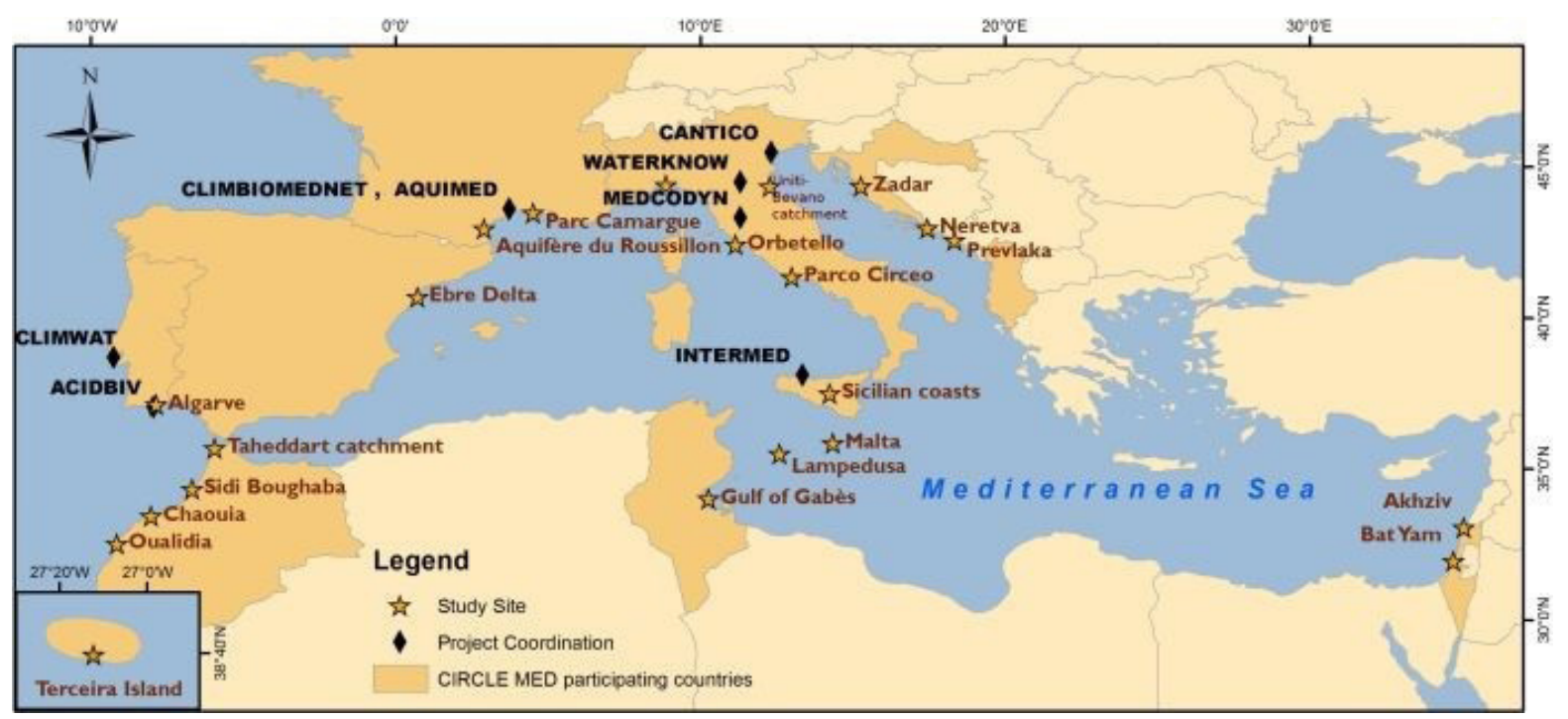

Figura 1: Área geográfica dos projetos e seus estudos de caso

Fonte: Site do Climate Impact Research Group for a larger Europe - Mediterranean Group 4

\section{Práticas daS EQUiPAS de INVESTIGAÇÃo RELATIVAS À INTERAÇÃo COM STAKEHOLderS}

\section{GRAU DE FORMALIZAÇÃo DA COLABORAÇÃo E INTERESSE NA DIMENSÃo SOCIAL}

Oficialmente, a chamada para projetos do Circle Med encorajava claramente a ligação entre os investigadores e os stakeholders (Circle Med, 2007; Circle2, 2011; Mojaisky, Leitner \& Martin, 2008). Contudo, nenhuma das propostas incluía stakeholders enquanto parceiros formais do projeto. Significa isto que, no início de cada projeto, a ligação com os stakeholders não era enquadrada institucionalmente, pelo que não existia nenhum protocolo de colaboração aprovado e assinado por ambas as partes. Em dois projetos, porém, a colaboração tornou-se efetiva através de relações já existentes com stakeholders externos. Num projeto, duas das equipas de investigação eram ONG's e noutro projeto um dos parceiros científicos era um consultor que podia interagir como um ator não científico. A tabela 2 (abaixo) apresenta informação relativa a cada projeto analisado.

\begin{tabular}{|c|c|c|c|}
\hline ACRÓNIMO & TíTULO DA INVESTIGAÇÃO & ОВJeTIVOS DA INVESTIGAÇÃO & PARCEIROS \\
\hline ACIDBIV & $\begin{array}{l}\text { Impactos integrados da acidificação ma- } \\
\text { rina, da temperatura e das mudanças nas } \\
\text { precipitações na biodiversidade costeira } \\
\text { dos bivalves e do pescado: como adaptar? }\end{array}$ & $\begin{array}{l}\text { Avaliação científica dos impactos da } \\
\text { acidificação nos bivalves. Propos- } \\
\text { tas de medidas de adaptação }\end{array}$ & 3 \\
\hline CANTICO & $\begin{array}{l}\text { Fatores antropogénicos locais e clima, } \\
\text { seus impactos na área costeira tunisina }\end{array}$ & $\begin{array}{l}\text { Avaliação dos riscos decorrentes de alterações } \\
\text { climáticas e dos impactos antropogénicos nas } \\
\text { zonas costeiras (suportes de apoio à deci- } \\
\text { são). Propostas de medidas de adaptação }\end{array}$ & 2 \\
\hline CLIMBIOMEDNET & $\begin{array}{l}\text { Influência da mudança climática na } \\
\text { biodiversidade, nos bens e nos ser- } \\
\text { viços das lagoas do Mediterrâneo }\end{array}$ & $\begin{array}{l}\text { Avaliação científica dos efeitos das alte- } \\
\text { rações climáticas nos ecossistemas das } \\
\text { lagoas e comparação destes com as al- } \\
\text { terações induzidas pelo Homem }\end{array}$ & 4 \\
\hline
\end{tabular}

\footnotetext{
${ }_{4}^{4}$ Retirado de www.circle-era.eu.
} 


\begin{tabular}{|c|c|c|c|}
\hline CLIMWAT & $\begin{array}{l}\text { Avaliação e monitorização do im- } \\
\text { pacto das alterações climáticas } \\
\text { nos recursos aquíferos costeiros e } \\
\text { nos ecossistemas dependentes }\end{array}$ & $\begin{array}{l}\text { Avaliação dos impactos das altera- } \\
\text { ções climáticas nos aquíferos costeiros } \\
\text { e nos ecossistemas dependentes }\end{array}$ & 2 \\
\hline INTERMED & $\begin{array}{l}\text { O impacto das alterações climáticas } \\
\text { nas comunidades intertidais no Me- } \\
\text { diterrâneo: perdas na integridade dos } \\
\text { ecossistemas costeiros e nos serviços }\end{array}$ & $\begin{array}{l}\text { Avaliação científica dos impactos das altera- } \\
\text { ções climáticas nos ecossistemas costeiros } \\
\text { e suas consequências socioeconómicas }\end{array}$ & 2 \\
\hline MEDCODYN & $\begin{array}{l}\text { Impactos das alterações climáti- } \\
\text { cas nos sistemas transicionais hi- } \\
\text { drológicos no Mediterrâneo }\end{array}$ & $\begin{array}{l}\text { Avaliação científica das vulnerabilidades } \\
\text { dos ecossistemas costeiros em relação às } \\
\text { modificações do clima e antropogénicas. } \\
\text { Propostas de medidas de adaptação. }\end{array}$ & 3 \\
\hline WATERKNOW & $\begin{array}{l}\text { Gestão integrada dos recursos hí- } \\
\text { dricos nas bacias costeiras: desafios } \\
\text { e estratégias de adaptação no âm- } \\
\text { bito das alterações climáticas }\end{array}$ & $\begin{array}{l}\text { Desenvolvimento de modelos integrados } \\
\text { para a gestão hídrica baseados em sistemas } \\
\text { de apoio à decisão incluindo os interes- } \\
\text { ses e as necessidades de stakeholders. }\end{array}$ & 3 \\
\hline
\end{tabular}

Tabela 2: Descrição dos projetos Circle: acrónimo do projeto, objetivos, e número de parceiros. Fonte: Site of the Climate Impact Research Coordination for a Larger Europe - Mediterranean Group.

Com base na análise documental facilitada pelos relatórios dos projetos, percebemos que a maior parte das propostas não considerava como prioridade a componente socioeconómica, não se obrigando minimamente a recrutar ou a incluir stakeholders nos projetos. Também as questões sociais acabaram por ter um papel secundário na maior parte dos projetos; eram-lhes atribuídos escassos ou nenhuns recursos humanos, bem como fundos muito limitados. Consequentemente, foi muito reduzido o tempo dedicado a objetivos deste tipo. A maior parte dos projetos versou sobre as dinâmicas dos ecossistemas relacionados com as alterações climáticas (por exemplo, o comportamento dos bivalves ou das espécies intertidais). Sempre que a dimensão socioeconómica esteve presente no estudo, ficou reduzida à análise de "bens e serviços oferecidos pelo ecossistema". Um investigador envolvido num destes projetos frisou esta ideia quando refletia sobre o seu próprio projeto de investigação:

O estudo das dimensões sociais não foi tido em conta no início do projeto, pois tratava-se de um projeto principalmente ecológico, criado por biólogos, em que os aspetos socioeconómicos eram periféricos. (Investigador)

Só um projeto conseguiu estabelecer uma colaboração com stakeholders, no sentido da co-produção de conhecimento. Esta ligação foi concretizada através da realização de workshops participativos. Neste caso, a equipa dedicou tempo, fundos e recursos humanos especializados (incluindo cientistas sociais) para esse trabalho. Outros projetos previram esta colaboração, mas os investigadores não a conseguiram efetivar, dado não a terem planeado suficientemente. Também não investiram nela nem tempo, fundos ou recursos humanos especializados. Finalmente, dois projetos que não tinham planeado atribuir importância à componente socioeconómica, acabaram por investir mais tempo e recursos na dimensão da colaboração, graças à ação de alguns membros da equipa e a algumas colaborações externas com um investigador da área das Ciências Sociais. 


\section{Principais objetivos E INTENÇões daS EQUiPAS DE INVESTIGAÇÃo}

Apesar das iniciativas no sentido de incluir os stakeholders na fase inicial dos processos de investigação serem limitadas, muitos investigadores mostraram-se preocupados com tal situação. Os investigadores entrevistados reconheceram a importância da relação com o stakeholder no sentido de recolher dados (por exemplo no caso de agentes da administração local) ou de aprender sobre o estudo de caso (por exemplo, com stakeholders locais tais como agricultores ou pescadores) ou até mesmo para, em conjunto com os decisores, serem criadas medidas de adaptação. Também consideraram importante difundir pelos stakeholders os resultados das pesquisas admitindo ser este um elemento chave para a melhoria da gestão dos ecossistemas estudados.

Algumas propostas indicavam especificamente a intenção de interagir com stakeholders. Outras propunham eventos, tais como workshops com intervenientes externos. Este foi o caso de três projetos: i) o projeto Acibdiv, em Portugal, no qual foram organizados workshops educacionais; ii) o projeto Medcodyn que, através de workshops, integrou cenários climáticos em modelos hidrográficos e iii) o projeto Climwat que contemplou a organização de eventos para a apresentação de resultados da investigação. Em alguns projetos contataram-se stakeholders, por ser preciso dialogar com gestores públicos de modo a obter informação (por exemplo, o Acidbiv Espanha). No entanto, os investigadores não desenvolveram iniciativas no sentido de contactar nem as comunidades locais, nem a sociedade civil. Os objetivos dos projetos não se centraram em alterações comportamentais quotidianas e, por conseguinte, os investigadores não precisaram de dados diretamente recolhidos junto dos cidadãos. Contudo, durante as entrevistas, todos os investigadores enfatizaram a importância que tem a ligação aos stakeholders para as suas pesquisas (mas também as dificuldades daí decorrentes).

A maior parte dos investigadores demonstrou também ter uma perceção positiva em relação ao conhecimento de outros intervenientes. Este dado é relevante, pois traduz a abertura para aprender com os stakeholders sobre as realidades locais, bem como a capacidade para partilhar conhecimentos considerados necessários à gestão dos recursos naturais. Alguns projetos revelaram desenvolver esforços no sentido de colaborar com stakeholders. Mas as opiniões dos membros dentro de um mesmo projeto de investigação dividiam-se. No projeto Acidbiv foi a própria administração local italiana que financiou um estudante de doutoramento para trabalhar diretamente com as cooperativas de pescadores. A maior parte dos investigadores entende que devem ser os próprios a comunicar os resultados das suas investigações:

Construímos um modelo de apoio à decisão. Tentámos integrar cenários de alterações climáticas e apresentá-los aos stakeholders. Neste projeto, não havia muita necessidade de interagir com os stakeholders. As interações serviam, sobretudo, para obter dados ou clarificar alguma questão, tais como o fornecimento de água pública, as previsões a longo prazo de consumo para a água potável e para a rega. (Investigador)

A colaboração com os stakeholders foi, muitas vezes, informal e não teve tradução nos objetivos ou nos resultados do projeto. $\mathrm{O}$ caso do Climbiomednet ilustra bem esta 
questão. Este projeto utilizou a metodologia de Dahlem. Esta é uma metodologia específica para desenvolver conhecimento entre peritos, convidando-se especialistas a contribuírem sobre um tema específico. Normalmente, esta metodologia não tem em conta a diversidade dos intervenientes envolvidos. No entanto, o projeto Climbiomednet convidou, de forma informal, alguns stakeholders para esta reunião de peritos. O responsável encarregue pelas reuniões referiu que a presença dos técnicos da Agência Costeira da Galiza era relevante para o problema em causa. No projeto Climwat também não houve nenhum acordo formal para trabalhar com a Agência Local para a Gestão da Água, mas as equipas de investigação de Portugal e de Marrocos trabalharam com técnicos das Agências de Gestão de Água, com empresas de água para consumo, em workshops e em visitas nos locais.

No caso francês do Medcodyn, o Instituto Tour du Valat organizou várias ações na região da Camarga para apresentar resultados científicos. No entanto, neste caso, o parceiro francês também estava envolvido em processos de gestão de recursos naturais em curso. Este Instituto é simultaneamente uma ONG e uma organização de investigação privada que defende a conservação das zonas húmidas do Mediterrâneo. Foi criada em 1954 e tem vindo a estabelecer ligações com outros stakeholders da região da Camarga (para uma informação mais pormenorizada sobre esta instituição, ver Dervieux, Jolly e Allouche, 2006). O comité executivo da Agência da Água do Parque Natural participou em workshops organizados durante o projeto Medcodyn.

O tipo de interação entre investigadores e stakeholders pode igualmente ser classificado de acordo com o tipo de resultados produzidos pelos projetos, como se pode constatar na tabela seguinte.

\begin{tabular}{|c|c|c|}
\hline Projecto & TIPO DE STAKEHOLDER & $\begin{array}{l}\text { TIPO DE INTERCÂMBIO E } \\
\text { SEUS RESULTADOS }\end{array}$ \\
\hline ACIDBIV PT & $\begin{array}{l}\text { Instituto Português do Mar e Atmosfera } \\
\text { Escolas secundárias }\end{array}$ & $\begin{array}{l}\text { Trabalho científico e dados científicos. Sessões } \\
\text { dedicadas a escolas secundárias no Algarve }\end{array}$ \\
\hline \multirow{2}{*}{$\begin{array}{l}\text { CLIMBiomednet Fr } \\
\text { CLIMBIOMEDNET SP }\end{array}$} & $\begin{array}{l}\text { Gestores de Recursos Naturais } \\
\text { e Institutos de Conservação. }\end{array}$ & $\begin{array}{l}\text { Através da internet, com um mapa } \\
\text { de informação climática }\end{array}$ \\
\hline & ONG Ambiental & $\begin{array}{l}\text { Participação em workshops Dahlem, troca } \\
\text { de pontos de vista e ideias com cientistas }\end{array}$ \\
\hline Climwatt PT & Administradores de Água, agricultores & $\begin{array}{l}\text { Encontros com o público com apre- } \\
\text { sentação de dados, em colabora- } \\
\text { ção com o projecto Aquimed }\end{array}$ \\
\hline $\begin{array}{l}\text { MEDCODYN Fr } \\
\text { MEDCODYN It } \\
\text { MEDCODYN MAR } \\
\end{array}$ & $\begin{array}{l}\text { Representantes de utiliza- } \\
\text { dores e instituições }\end{array}$ & Reuniões regulares e workshops \\
\hline
\end{tabular}

Alguns projetos desenvolveram atividades com estudantes e professores em escolas secundárias. A equipa de investigação portuguesa do projeto Acidbiv já contemplava um programa chamado "Cientistas na escola", através do qual os investigadores trabalhavam anualmente com as escolas públicas. Os resultados científicos do projeto 
Acidbiv foram integrados neste programa e os investigadores do projeto Acidbiv apresentaram os dados científicos em mais de 15 escolas do Algarve. O projeto Climbiomednet organizou, através da internet, uma interação indirecta com o público ou com intervenientes específicos como pescadores, com informações climáticas. No âmbito do mesmo projeto, um profissional de uma Instituição da Água foi convidado para participar em workshops organizados em Espanha. Outros projetos aplicaram questionários a stakeholders, mas o nível de respostas foi baixo (projeto Cantico).

No projeto Medcodyn, a interação com as autoridades administrativas foi direta. Contudo, como descrito pelo parceiro francês, esta interação fazia-se sob a forma de reuniões informais em que se discutiam e validavam dados para medidas de adaptação. Só o projeto Medcodyn utilizou a metodologia de workshops como espaço para debate com diferentes intervenientes ligados à mesma problemática.

\section{Modelos DE INTERAÇÃo}

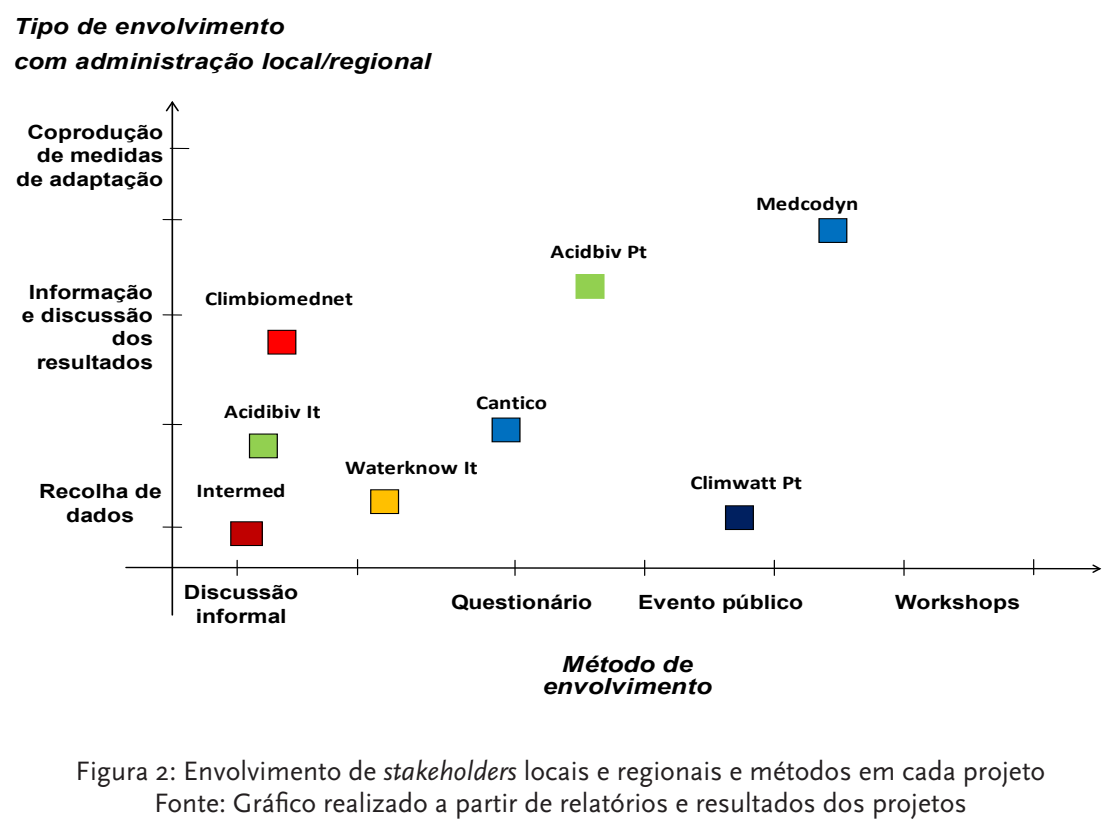

O tipo de interação entre os investigadores e a administração local ou a sociedade civil pode ser também classificada com base nos métodos utilizados para interação e nos objetivos que se pretende atingir (Bento et al., 2013). Os métodos utilizados para interagir podem ser reuniões informais, questionários, eventos públicos ou até workshops participativos.

A figura acima sumariza o nível de interação dos stakeholders no trabalho de investigação, bem como os diferentes métodos utilizados para os envolver nos projetos. O grau de envolvimento pode incluir investigadores que pedem e reúnem dados dos stakeholders ou stakeholders que colaboram com os investigadores na coprodução de medidas de adaptação para as alterações climáticas. A maior parte dos projetos só revela a preocupação em reunir e trocar dados com os stakeholders. O projeto Intermed 
preocupou-se com o impacto das alterações climáticas nas comunidades intertidais, mas esperava-se que o projeto tivesse alguma dimensão social e económica na medida em que o estudo iria analisar o impacto do aumento de temperaturas na produção das espécies. Este impacto foi essencialmente analisado do ponto de vista dos ecossistemas naturais e os stakeholders contribuíram pouco durante a investigação. Investigadores de outras equipas realizaram entrevistas informais a pescadores, movimentos ecologistas e administrações dos recursos naturais. Disseram-nos, de modo geral, que a relação com os stakeholders era complexa e difícil porque havia interesses opostos.

Torna-se claro que nenhum projeto de investigação levou a colaboração ao mais alto nível - coprodução de conhecimento - o que implica a participação dos stakeholders numa reflexão sobre o desenho do projeto, a escolha de métodos, os objetivos do projeto, a análise de dados e a disseminação dos resultados. Só num dos projetos se conseguiu co-produzir medidas de adaptação baseadas numa discussão alargada entre administradores e utilizadores. Este resultado provou que um processo de investigação que inclua stakeholders, abrangendo diferentes formas de conhecimento, seria bastante mais complexo do que algumas equipas de investigação inicialmente imaginavam.

Podemos observar, igualmente, que os métodos utilizados podem variar quanto à intensidade do envolvimento pretendido. Assim, os métodos usados incluem uma série de possibilidades desde a situação em que exigem pouco esforço organizativo e diminuto empenho por parte do stakeholder como a situação em que exigem um grande esforço organizativo e solicitam uma intervenção exigente para o stakeholder. Assim, a lista de métodos usados começa com situações em que existe menos troca, o que equivale a uma simples interação e discussões informais que permitem alguma troca de informação. Os 3 outros métodos consistem em formas mais estruturadas. $O$ primeiro, o questionário, é uma forma indireta de envolvimento, centrada essencialmente em conhecimento específico, que foi utilizado para interagir com os stakeholders. Os últimos métodos são dialógicos, uma vez que implicam um contato direto e uma troca de conhecimentos, tanto em eventos públicos como em workshops participativos. Uma vez mais, os métodos que implicaram menos solicitação e colaboração foram os mais utilizados.

O projeto Acidbiv foi um dos que usou o questionário para obter informação. O parceiro português organizou algumas sessões para divulgar em escolas secundárias dados gerais sobre bivalves e clima. O objetivo era mais disseminar informação do que discuti-la. No projeto Climwat, a equipa portuguesa, em parceria com o Aquimed, organizou uma sessão pública na qual os resultados do projeto foram apresentados à população. Esta sessão decorreu num espaço público da região onde o estudo foi desenvolvido. Foi levada a cabo para substituir uma sessão mais participativa que estava na proposta inicial e em que se previa que os stakeholders expusessem os seus conhecimentos, assim como um modelo de apoio à decisão para uso do aquífero local. No projeto Medcodyn, houve várias reuniões com diversos stakeholders. No caso francês do Medcodyn não se organizaram workshops específicos, mas os resultados do projeto foram discutidos com uma comissão de gestão formal do Parque Natural da Camargue.

Finalmente, quando comparamos o envolvimento da equipa de investigação segundo o tipo de stakeholder, verifica-se que o grau de interação com stakeholders como 
instituições públicas foi maior do que o grau de envolvimento com stakeholders da sociedade civil. Em primeiro lugar, a relação com pessoas da administração era, na maioria dos casos, obrigatória para os projetos mencionados. Os investigadores precisavam de dados para a sua investigação. Em segundo lugar, esta interação era também descrita como sendo mais "fácil", pois o pessoal da administração é normalmente mais qualificado e usa a terminologia técnica, criando um ambiente mais propício à colaboração (Saner, 2007). A colaboração é também mais facilitada pois, normalmente, os investigadores e os administradores pertencem à mesma rede social, encontrando-se, assim, com frequência em locais comuns tais como comissões, reuniões, conferências e eventos sociais ligados à água ou aos recursos costeiros. Este facto não pode ser desvalorizado. Como vários autores mostraram, o papel das relações pessoais em comunidades epistémicas de diferentes contextos - tais como laboratórios (Jasanoff, 1996) ou empresas (Mercklé, 2004) - é um elemento crucial para a colaboração. Contudo, não devemos ignorar que esta colaboração se limita, a maior parte das vezes, a uma troca de informação técnica. Quer isto dizer que este tipo de aliança não abre, necessariamente, portas para uma discussão sobre criação de políticas específicas de adaptação às alterações climáticas, tal como se pode verificar na figura abaixo.

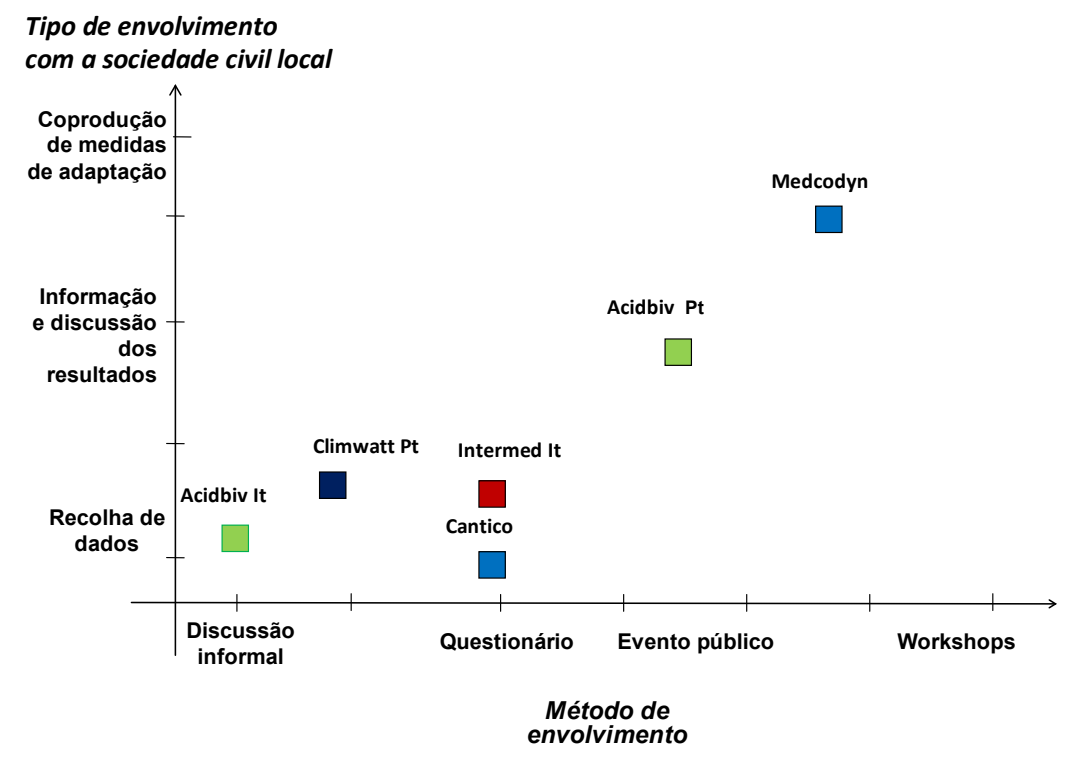

Figura 3: Envolvimento de stakeholders da sociedade civil e métodos usados em cada projeto Fonte: Gráfico realizado a partir de relatórios e resultados dos projetos

Verificando a escolha de modelos de interação com intervenientes locais nos projetos Circle, nota-se uma utilização mais frequente de métodos de envolvimento com o stakeholder que são menos exigentes para ambos em termos de tempo, de energia, de conhecimento e de apoio financeiro. As relações entre investigadores e stakeholders basearam-se na mera troca de informação, o que é insuficiente para produzir conhecimento relativo à gestão da sustentabilidade dos recursos, uma vez que tal requer um esforço científico integrado. 


\section{CONCLUSÕES}

Normalmente, a ciência é produzida pelos cientistas, a definição dos objetivos científicos é feita pelos académicos; a única coisa que os cientistas fazem em relação a outros é entregar aos stakeholders, que pertencem a outro mundo, o seu produto. Na verdade, as coisas não deviam ser assim; um ou mais stakeholders deveriam estar dentro "da caixa" da ciência, todos numa mesa redonda. De outra forma, é demasiado simplista. (Investigador entrevistado)

Os relatórios dos projetos e as entrevistas com os investigadores mostram que os projetos ao abrigo do programa Circle responderam de forma diferente à chamada para o estabelecimento de uma colaboração com os stakeholders. Os projetos do Circle-Med diferiram também quanto ao tipo de stakeholder que escolheram - alguns optaram por ambos os tipos (administração local e regional e sociedade civil), mas a maioria escoIheu um só tipo, e outros não escolheram nenhum tipo de stakeholder. Podemos dizer que, em geral, a interação entre investigadores e stakeholders decorreu de forma redutora e oportunista. As experiências efetivas de interação foram raras e ficaram longe dos objetivos enunciados na chamada de projetos.

Além do mais, os investigadores entrevistados foram unânimes na referência às dificuldades enfrentadas na ligação com os stakeholders. Os investigadores entrevistados apontaram como principais razões a curta duração dos projetos (24 meses) e o baixo financiamento. Dadas estas limitações, todos os coordenadores admitiram ser extremamente difícil atingir, ao mesmo tempo, os objetivos científicos e de colaboração com os stakeholders. A falta de financiamento foi já identificada como sendo um obstáculo nos projetos de investigação colaborativos (Cummings \& Riesler, 2005). Quanto às limitações de tempo, só os projetos com um trabalho prévio de relacionamento com stakeholders, e pessoas da equipa especializadas neste domínio, é que conseguiram ter sucesso. Podemos observar que as "ligações fracas" com stakeholders não foram exploradas: somente os projetos que tinham relações mais antigas e de confiança tiveram condições para pôr em prática esta colaboração.

Todos estes factos explicam a minimização da possibilidade de juntar mundos tão diversos como a ciência, a administração e os cidadãos, no sentido da capacitação da aprendizagem coletiva (Bradshaw \& Borchers, 2000). Os resultados deste estudo reafirmam existir ainda um longo caminho a percorrer para que se chegue a uma ciência verdadeiramente integrada e colaborativa aproximando cientistas e stakeholders. Uma ciência que integre vários parceiros, investigadores das Ciências Naturais e sociais e não-cientistas, tais como a administração ou stakeholders locais tem de diversificar os meios e os espaços de troca de conhecimentos (Barash, 2005). A atual organização da ciência e das instituições públicas constitui, evidentemente, um obstáculo a esta situação. No entanto, a falta de cooperação e de colaboração entre cientistas e não-cientistas tem também de se enquadrar num contexto científico em que as Ciências Sociais e as Ciências Naturais estão separadas. Com efeito, a interdisciplinaridade faz parte, sem 
dúvida, do desafio que é de desenvolver uma ciência que abarque tanto a dimensão natural como a social dos recursos naturais, indo ao encontro de soluções sustentáveis (Brandt et al., 2013; Cummings \& Riesler, 2005; Lowe \& Phillipson, 2009).

Achamos que a reflexão sobre estes tópicos será um primeiro passo. Groot e outros (2014) chamam a atenção para a necessidade de trocar experiências e fazer pontes de conhecimento, ao realizar programas para adaptação climática. Mas tal deve ser acompanhado de alterações a nível das políticas vigentes que definem o desempenho científico e os incentivos para a carreira de investigação. As atuais políticas para a ciência reservam baixos orçamentos para a investigação. A avaliação é centrada em indicadores de publicações científica e não se dá atenção a questões de transferências de conhecimento. Também para as administrações, as atividades são consideradas no plano imediato de curto prazo. Todos estes factores dissuadem abordagens colaborativas. É preciso haver uma maior flexibilidade (orçamental, por exemplo, mas também organizacional) e uma maior abertura entre atores, bem como uma melhor distribuição de poder por todos os parceiros no processo científico. Havendo abertura, humildade e vontade (por parte da ciência), haverá certamente suficiente conhecimento acumulado para operar uma transformação em ambos os domínios e para juntar dois mundos que estão há muito separados.

Apresentamos, de seguida, algumas recomendações com vista a melhorar a colaboração entre cientistas e stakeholders na investigação sobre alterações climáticas, no que respeita ao conteúdo das chamadas de propostas científicas, à sua monitorização e avaliação final. As chamadas para projetos deverão usar critérios específicos no que diz respeito a colaboração com outros parceiros. Esta colaboração tem de estar definida e formalizada na proposta, com a apresentação dos intervenientes e dos seus interesses e responsabilidades na investigação. Há mais fatores a ter em consideração nas chamadas: as questões dos prazos, da logística para a colaboração e até do co-financiamento por parte dos stakeholders não ligados à ciência. Com vista a uma melhor colaboração, devia também equacionar-se a etapa de envolvimento dos stakeholders. Estes deviam ser envolvidos ao longo do processo de investigação podendo, igualmente, influenciar o processo de investigação ou as políticas de planeamento (Lang et al., 2012; Hauck, 2015; Reed, 2009).

Os financiadores deveriam definir incentivos às equipas de investigação em função do envolvimento dos stakeholders; este incentivo poderia ser explicitado na chamada para projetos. Assim, equipas cujas propostas apresentassem altos níveis de colaboração com stakeholders, seriam alvo prioritário do financiamento. Podem ser estabelecidos critérios claros e objetivos (tipo de stakeholder, resultados da colaboração, calendário da colaboração, metodologias usadas, fase da investigação, recursos financeiros dispensados). Os programas de investigação poderiam também ter em conta a troca de conhecimentos resultantes da colaboração, com avaliações regulares junto das equipas de stakeholders e de investigadores. Esta avaliação seria um instrumento para monitorizar o nível e o impacto da colaboração entre stakeholders e equipas científicas e serviria, ao mesmo tempo, para fazer a ponte entre o programa de investigação e os stakeholders, 
particularmente decisores. Seria necessário um maior envolvimento entre o Programa Circle e os Ministérios do Ambiente dos vários países e as agências de financiamento.

Os programas podem também definir a colaboração tendo em vista uma integração entre Ciências Sociais e Ciências Naturais, adoptando, para isso, propostas de investigação com uma aproximação à interdisciplinaridade. A interdisciplinaridade pode ser implementada através da participação de cientistas sociais e da integração de problemáticas sociais entendidas de forma ampla, isto é, integrando o ponto de vista cultural, psicológico, social e histórico destas questões. Na nossa perspetiva, os programas de investigação têm de estimular a transferência de informação, ideias, diferentes conhecimentos através de vários canais, incluindo metodologias participativas, debates públicos, redes formais e informais. A acrescentar, o relatório final de avaliação do projeto feito pelos financiadores deveria considerar seriamente o desencontro entre os resultados e os objetivos pretendidos no que diz respeito à interação entre cientistas e stakeholders.

\section{REFERÊNCIAS BIBLIOGRÁFICAS}

Barash, D. (2005). C.P. Snow: Bridging the two-cultures divide. The Chronicle Review, 52 (14), 10-15.

Bento, S.; Richard-Ferroudji, A.; Varanda, M.; Faysse, N. \& Rosa., A. (2013). Final Report: Taking Stock of the CIRCLE-MED Program Experience. Communication Between Scientists and Stakeholders Regarding Adaptation to Climate Change.

Bradshaw, G. A. \& Borchers, J.G. (2000). Uncertainty as information: narrowing the science-policy gap. Conservation Ecology, 4(1), 7. Retirado de http://www.consecol.org//ournal/vol4/iss1/art7.

Brandt, P.; Gralla, F.; Luederitz, C.; Lang, D.J;; Newig, J.; Reinert, F.; Abson,D. \& Wehrden, V.H. (2013). A review of transdisciplinary research in sustainability science. Ecological Economics, 92, 1-15.

Circle-Med (2007). First Coordinated Call: Integrated Coastal Zones and Water Management. Retirado de http:// www.circle-med.net/.

Circle 2. Climate Impact Research \& Response Coordination for a Larger Europe - EUFP7-ERA-NET. (2011). Proceedings Circle Med Final Conference. Aix-en-Provence. Retirado de http://circle-med.net/index. php?pagename=researchcall.

Cohen, S. J. (1997). Scientist-stakeholder collaboration in integrated assessment of climate change: lessons from a case study of Northwest Canada. Environmental Modeling and Assessment, 2, 281-293.

Comissão Europeia. (2014). Climate Change (Special Eurobarometer 409). Bruxelas.

Forbes, S. (1925). The Lake as a Microcosm. Illinois Natural History Survey Bulletin, 15, 537-550.

Cummings, J. \& Riesler, S. (2005). Collaborative research across disciplinary and organizational boundaries. Social Studies of Science, 35(5), 704-722.

Dervieux, A.; Jolly, G. \& Allouche, A. (2006). Gestion de l'eau et projet de territoire : vers une gestion intégrée du delta du Rhône. Vertigo - La Revue Électronique en Sciences de l'Environnement, 7(3). Retirado de http://vertigo.revues.org/1908; DOI: 10.4000/vertigo.1908.

Driessen, P.; Leroy,P. \& Vierssen,V. (2010). From climate change to social change: Perspectives on science-policy interactions. Utrecht: International Books. 
Eakin, H.; Magaña, V.; Smith J.; Moreno J. L.; Martínez J. M. \& Landavazo, O. (2007). A stakeholder drivenprocess to reduce vulnerability to climate change in Hermosillo, Sonora, Mexico. Mitigation and Adaptation Strategies for Global Change, 12, 935-955.

Funtowics, S. O. \& Ravetz, J. R. (1990). Uncertainty and quality in science for policy. Amsterdam: University Press.

Grimble, R. (1998). Stakeholder methodologies in natural resource management. Socio-economic methodologies. Best Practice Guidelines. Chatham: Natural Resources Institute.

Groot, A.; Hollaender, K. \& Swart, R. (2014). Productive science-practice interactions in climate change adaptation. Lisboa: Faculdade de Ciências.

Grundman, R. \& Stehr, N. (2012). Lever-tracy climate change: what role for sociology? A response to constance. Current Sociology, 58(6), 897-910.

Hauck, J.; Stein, C.; Schiffer, E. \& Vandewalle, M. (2015). Seeing the forest and the trees: Facilitating participatory network planning in environmental governance. Global Environmental Change, 35, 400-410.

Hoverman, S.; Ross, H.; Chan, T. \& Powell, B. (2011). Social learning through participatory integrated catchment risk assessment in the Solomon Islands. Ecology and Society, 16(2), 17. Retirado de http:// www.ecologyandsociety.org/vol16/iss2/art17/.

Hulme, M.; Dessai, S.; Lorenzoni, I. \& Nelson, D. (2009). Unstable climates: Exploring the statistical and social constructions of normal climate. Geoforum, 40, 197-206.

IPCC. Intergovernmental Panel on Climate Change. (2007). Summary for Policymakers. In S. Solomon; D. Qin; M. Manning; Z. Chen; M. Marquis; K. B. Averyt; M. Tignor \& H. L. Miller (Eds.), Climate Change 2007: Mitigation of Climate Change. Working Group III Contribution to the Fourth Assessment Report of the Intergovernmental Panel on Climate Change (pp. 1-18). Cambridge and New York: Cambridge University Press.

Irwin, A. (1995). Citizen science: a study of people, expertise and sustainable development. Londres: Routledge.

Jasanoff, S. (1996). Is Science Socially Constructed-and Can It Still Inform Public Policy? Science and Engineering Ethics, 2(3), 263-276.

Katz, J. S. \& Martin, B. R. (1997). What is research collaboration? Research Policy, 26, 1-18.

Kuper, M.; Dionnet, M.; Hammani, A.; Bekkar, Y.; Garin, P. \& Bluemling, B. (2009). Supporting the shift from state water to community water: lessons from a social learning approach to designing joint irrigation projects in Morocco. Ecology and Society, 14(1), 19. Retirado de http://www.ecologyandsociety.org/vol14/ iss1/artig/.

Lang, D.; Wiek, A.; Bergmann, M.; Stauffacher, M.; Martens, P.; Moll, P.; Swilling, M. \& Thomas, C. (2012). Transdisciplinary research in sustainability science: practice, principles and challenges. Sustainability Science, $7(1), 25-43$.

Latour, B. (2014). War and peace in an age of ecological conflicts. Revue Juridique de l'Environnement, 39(1), $51-63$.

Lovens, A.; Turkelboom, F.; Demeyer, R.; Garcia-Llorente, M.; Hauck, J.; Kelemen, E.; Teng, C.; Tersteeg, J.; Lazányi, O.; Martin-Lopez, B.; Pataki, G. \& Schiffer, E. (2015). Openness manual: stakeholder analysis for environmental decision-making at local level. Publication developed in the framework of OpenNESS (FP7 Project). INBO, Brussels, Belgium. 
Lowe, P. \& Phillipson, J. (2009). Barriers to research collaboration across disciplines: scientific paradigms and institutional practices. Environment and Planning A, 41(5), 1171-1184.

Mercklé, P. (2004), Sociologie des réseaux sociaux. Paris: La Découverte.

Mojaisky, M.; Leitner, M. \& Martin, D. (2008). Minutes of circle med call kick-off meeting. Retirado de http:// circle-med.net/index.php?pagename=researchcall.

Nowotny, H.; Scott, P. \& Gibbons, M. (2003). Mode 2 revisited: The new production of knowledge. Minerva, 41, 179-194.

Phillipson, J.; Lowe, P.; Proctor, A. \& Ruto, E. (2012). Stakeholder engagement and knowledge exchange in environmental research. Journal of Environmental Management, 95, 56-65.

PNUD, Programa das Nações Unidas para o Desenvolvimento. (2007-2008). Relatório de Desenvolvimento Humano 2007/2008. Combater as Alterações Climáticas: Solidariedade Humana num Mundo Dividido. Retirado de http://hdr.undp.org/en/content/human-development-report-20078.

Ravetz. J. (1999). Post-normal science: an insight now maturing. Futures, 31(7), 641-646.

Richard-Ferroudji, A. (2011). Limites du modèle délibératif : Composer avec différents formats de participation. Politix, 24(96), 161-181.

Reed, M. S. (2008). Stakeholder participation for environmental management: A literature review. Biological Conservation, 141 (10), 2417-2431. doi: 10.1016/j.biocon.2008.07.014.

Reed, M.; Graves, A.; Dandy, N.; Posthumus, H.; Hubacek, K.; Morris, J.; Prell, C.; Quinn, C. \& Stringer, L. (2009). Who's in and why? A typology of stakeholder analysis methods for natural resource management. Journal of Environmental Management, 9o(5), 1933-1949.

Saner, M. (2007). A map of the interface between science and policy. Ontario: Council of Canadian Academies.

Santos, F.D. (2012). Alterações globais. Os desafios e os riscos presentes e futuros. Lisboa: Fundação Francisco Manuel dos Santos.

Schiermeier, Q. (2007). Get pratical, urge climatologists. Nature, 448, 234-235.

Schneider, D. (2000). Local knowledge, environmental politics, and the founding of Ecology in the United States: Stephen Forbes and the lake as a microscom. Isis, 91(4), 681-705.

Von Korff, Y.; Daniell, K.A.; Moellenkamp, S.; Bots, P. \& Bijlsma, R.M. (2012). Implementing participatory water management: Recent advances in theory, practice and evaluation. Ecology and Society, 17(1), 30. Retirado de http://www.ecologyandsociety.org/volı/issı/art3o/.

Wilby, R. \& Dessai, S. (2010). Robust adaptation to climate change. Weather, 65(7), 180-185.

Wynne, B. (1996). May the sheep safely graze? A reflexive view of the expert-lay knowledge divide. In S.Lash, B.Szerszynski \& B.Wynne (Eds.), Risk, Environment and Modernity: Towards a New Ecology (pp. 44-83). Londres: Sage.

\section{FinANCIAMENTo}

Este artigo foi realizado no âmbito do projeto científico intitulado "Taking stock of the CIRCLE-MED Program experience: Communication between scientists and 
stakeholders regarding adaptation to climate change" (CIRCLE-MED/0001/2011) financiado pelo Pograma Circle-Med, o Ministério Francês da Ecologia, Desenvolvimento Sustentável, Transportes e Habitação e pela Fundação para a Ciência e Tecnologia (FCT).

\section{NOTAS BIOGRÁFICAS}

Sofia Bento é socióloga e professora auxiliar no Instituto Superior de Economia e Gestão da Universidade de Lisboa (ISEG-UL). É atualmente investigadora do SOCIUS, unidade especializada em Sociologia pertencente ao Centro de Investigação em Ciências Sociais e Gestão (CSG) do ISEG-UL. É doutorada em Sociologia da Inovação pela École Nationale Supérieure des Mines de Paris. Pesquisa nos estudos sociais da ciência nomeadamente em temas como o clima, a água, o envolvimento e a participação dos cidadãos em políticas ambientais.

E-mail: sbento@iseg.ulisboa.pt

CSG. Centro de Investigação em Ciências Sociais e Gestão, ISEG-Instituto Superior de Economia e Gestão, Rua Miguel Lupi, 20, 205, 1249-078 Lisboa-Portugal

Marta Pedro Varanda é socióloga. É atualmente professora auxiliar no Instituto Superior de Economia e Gestão da Universidade de Lisboa (ISEG-UL) e investigadora no SOCIUS/CSG. É doutorada em Sociologia pela Université de Lille I (2003) e tem um mestrado em Sociologia das Organizações da University of South Carolina (1991) e uma licenciatura em Sociologia e Economia (1989).

Endereço eletrónico: marta@iseg.ulisboa.pt

CSG. Centro de Investigação em Ciências Sociais e Gestão, ISEG-Instituto Superior de Economia e Gestão, Rua Miguel Lupi, 20, 205, 1249-078 Lisboa-Portugal

Audrey Richard-Ferroudji é engenheira e é doutorada em sociologia. Trabalhou 11 anos no IRSTEA (ex-CEMAGREF), um instituto de engenharia ambiental e agronómica, inserido na unidade de investigação G-EAU em Montpellier. O seu trabalho de investigação centra-se na governança da água e na gestão participativa. Integrou a equipa que concebeu e levou a cabo workshops prospetivos e participativos junto de agricultores, no quadro de estratégias de adaptação para a gestão de aquíferos no contexto de escassez de água na região mediterrânica (Aquimed project, Era-Net Circle).

E-mail: audrey.richard@ifpindia.org

French Institute of Pondicherry, 11, Saint Louis Street, Pondicherry 605 001, India.

Nicolas Faysse é cientista social na unidade mixta Gestão de Recursos Hídricos, Atores e Usos da Água no Centro de Investigação e Desenvolvimento da Agronomia (CIRAD) em França. É doutorado em Economia Institucional pela Université Paris 10. Encontra-se atualmente a desenvolver a sua atividade de investigação no Instituto Asiático de Tecnologia na Tailândia.

E-mail: nicolas.faysse@cirad.fr 
School of Environment, Resources and Development, P.O. Box 4, Khlong Luang, Pathumthani 12120, Thailand.

* Submetido: 22-10-2015

* Aceite: 09-11-2015 